\title{
Editorial: The Impact of COVID-19 on Sustainable Consumption
}

\author{
Krystal M. Perkins ${ }^{1 *}$, Luis Velazquez ${ }^{1}$ and Nora Munguia ${ }^{2}$ \\ ${ }^{1}$ Departamento de Ingeniería Industrial, Universidad de Sonora, Hermosillo, Mexico, ${ }^{2}$ University of Sonora, Hermosillo, \\ Mexico
}

Keywords: sustainable consumption, COVID-19, sustainable development goals, sustainable assessment, behavior

\section{Editorial on the Research Topic}

\section{The Impact of COVID-19 on Sustainable Consumption Behaviors}

The Sustainable Development Goals (SDG) set the year 2030 as the endpoint by which major global and social environmental crises should have been tackled. A noteworthy feature of the SDGs is that many relate to responsible consumption, with SDG 12 directly involving sustainable consumption. The Sustainable Development Goal 12 states: "to ensure sustainable consumption and production practices necessarily entails to respect the biophysical boundaries of the planet and to reduce current global consumption rates in order to fit with the biophysical capacity to produce ecosystem services and benefits" (United Nations Environment Program, 2020). The COVID19 global pandemic has drastically shifted the landscape of sustainable consumption, and the anticipated future impacts are not well-known. Moved by this uncertainty, the editorial team proposed the Research Topic The Impact of COVID-19 on Sustainable Consumption and sought work from scholars that bring together transdisciplinary knowledge of sustainable consumption that intersect with the contextual factors, conditions, and challenges encompassing the COVID-19 pandemic. The contributors to this Research Topic represent an expansive breadth and originality of questions, perspectives, and research designs that excavate significant understandings of sustainable consumption. Additionally, the contributions are empirical and conceptual and include scholars across national and disciplinary borders. This range in topics is intended to establish and provoke new epistemologies and avenues for future research. Turning specifically to the published manuscripts, four distinct elements/themes stand out: multidisciplinary perspectives, embedded contexts, the psychological and the sustainable, and the potential of sustainable consumption in a COVID-19 world. In what follows, a brief overview of this Research Topic's contributions organized by the four elements/themes is provided.

An important element that runs across the manuscripts is their application of multidisciplinary perspectives. For example, Giannetti et al. establishes and quantifies an integrative framework of environmental sustainability. They propose eight different combinations of sustainability scenarios, where the ideal scenario, Paradise, is one where there is "low pressure on the natural environment, high productivity, and happiness" (p. 5). The strength of Giannetti's et al. analysis is their integration of perspectives typically found in scholarly sustainability circles (e.g., ecological footprint) and ones that are less well-known (e.g., happiness). In their conceptual paper, Perkins et al. also engage the theme of sustainable consumption in the context of COVD-19 by discussing two underutilized and rarely drawn upon bodies of work: supply chain sustainability and postcolonial theory. Perkins's et al. contribution brings attention to the limitations of sustainable consumption approaches, practices, and theoretical perspectives and makes clear that a deep commitment to social justice needs to be rooted in discourses of sustainable consumption. The 
frameworks introduced by Giannetti et al. and Perkins et al. hold great promise for future research.

The manuscripts for this Research Topic also draw theoretically and empirically on questions that emerge at the borders of social context. Specifically, each manuscript probes the social and national contexts of the COVID-19 pandemic that shape and intersect with sustainable consumption across varied regions globally, like Italy and Germany, two nations that faced very different pandemics. For example, Rovetta's paper evaluates how COVID-19 restrictions impact particulate matter concentrations and influence everyday Italian citizen's consumption habits at both the local and national levels. Rovetta found that lockdowns did not reduce particulate matter consumption, but did alter consumption habits. Similarly, Reusswig et al. compare the carbon footprint of a voluntary "real lab" situation (e.g., 100 private households in Berlin from 2018) to the carbon footprint of an involuntary "real lab" situation imposed by the COVID-19 lockdowns. They found reductions in both voluntary and imposed household lab situations. Collectively, this body of work spotlights the need to be attuned to specific social contexts in consideration of sustainable consumption.

A third distinct element of this Research Topic is its elaboration of the human behavioral and psychological linkages to sustainable consumption. A human behavioral dimensions perspective of sustainable consumption has been in development for several decades (see Ruby et al., 2020) and several contributors to this Research Topic are paradigmatic examples of this work. Two of the empirical contributions, for example, explore consumption behavioral changes imposed by COVID-19 lockdowns (Reusswig et al.; Rovetta). Perkins et al. also propose that the focus of sustainable consumption should broaden to include collective ethics. Finally, Giannetti's et al. perspective

\section{REFERENCES}

Ruby, M. B., Walker, I., and Watkins, H. M. (2020). Sustainable consumption: the psychology of individual choice, identity, and behavior. J. Soc. Issues 76, 8-18. doi: 10.1111/josi.12376

United Nations Environment Program (2020). Ensure Sustainable Consumption and Production Patterns. Available online at: https://www.unenvironment. org/explore-topics/sustainable-development-goals/why-do-sustainabledevelopment-goals-matter/goal-12

Conflict of Interest: The authors declare that the research was conducted in the absence of any commercial or financial relationships that could be construed as a potential conflict of interest. piece maintains that environmental sustainability and reaching the Paradise scenario are partially psychological matters. These studies are demonstrating that many aspects of sustainable consumption are inherently psychological. Specifically, this work suggests that scrutinizing beliefs, concerns, social relations, and ideologies related to sustainable consumption is paramount to earnest change.

To conclude with the obvious, the contributions to this topic issue illustrate the potential of sustainable consumption in our post-COVID-19 world, but with some important caveats. Perkins et al., for example, urge for practices like supply chain sustainability to be adopted more broadly beyond our post-pandemic world. Likewise, Giannetti et al. assert that the COVID-19 crisis represents a rare, but narrow, window of opportunity to bring about a Paradise scenario, but can only be achieved by changing our current business-as-usual productionconsumption mentality. At the broadest level, Rovetta proposes that the COVID-19 pandemic has demonstrated the need for structural changes and governmental actions related to sustainable consumption. Finally, several contributors note that the potential of sustainable consumption in our postCOVID-19 world rests on the field moving away from longstanding concepts, theories, and perspectives that exclusively rely on the management of limited resources (Giannetti et al.; Perkins et al.). We hope that these contributions challenge researchers to probe new questions and serve as a model for future research.

\section{AUTHOR CONTRIBUTIONS}

KP: conceptualization and writing-original draft. KP and LV, and NM: review and editing. All authors contributed to the article and approved the submitted version.

Publisher's Note: All claims expressed in this article are solely those of the authors and do not necessarily represent those of their affiliated organizations, or those of the publisher, the editors and the reviewers. Any product that may be evaluated in this article, or claim that may be made by its manufacturer, is not guaranteed or endorsed by the publisher.

Copyright (c) 2021 Perkins, Velazquez and Munguia. This is an open-access article distributed under the terms of the Creative Commons Attribution License (CC BY). The use, distribution or reproduction in other forums is permitted, provided the original author(s) and the copyright owner(s) are credited and that the original publication in this journal is cited, in accordance with accepted academic practice. No use, distribution or reproduction is permitted which does not comply with these terms. 